\title{
Attractiveness of an Interactive Public Art Installation
}

\author{
Jun $\mathrm{Hu}^{1}$, Duy Le ${ }^{1}$, Mathias Funk ${ }^{1}$, Feng Wang ${ }^{1,2}$, and Matthias Rauterberg ${ }^{1}$ \\ ${ }^{1}$ Department of Industrial Design, Eindhoven University of Technology, The Netherlands \\ ${ }^{2}$ School of Digital Media, Jiangnan University, P.R. China \\ $\{j . h u, 1$. duy , m. funk, feng.wang, g.w.m.rauterberg\} atue.nl
}

\begin{abstract}
Interaction experiences with public art installations are becoming ubiquitous recently, however, interaction is usually unidirectional and the actual experience not very rich. This work reports on an interactive public art installation aiming at increasing the level of social connectedness among visitors, and the results of evaluating the attractiveness of the installation. By connecting visitors and computers physiologically, the installation has clear impact on social interaction and it also shows the attractiveness to people from aspects such as creativity, novelty, inviting and motivating. In this work we also found that the AttrakDiff instrument to be useful and convenient in evaluating the attractiveness of public art installations.
\end{abstract}

Keywords: Interactive Installation, Public Art, Attractiveness, Social Connectedness, Computers as Social Actors.

\section{Introduction}

The advances in science and technology bring the public digital arts from traditional media towards new media types often enabled by recent technological developments. The use, the language and the implications of the material itself are different from it being applied as a carrier for public digital arts. These new media types of public digital arts are in need of new carriers and new form languages for its progress and prosperity in the age of the new technologies, from traditional ones with static forms to new ones with dynamic and interactive forms [1].

Public interactive art installations are effective in addressing and engaging multiple people, and displays and projections are often used for these installations as output devices. While the use for advertisements, entertainment and promotion is quite farspread, the usual modus operandi of a single projection is to engage people as a single person in a 1:1 message. One of the drawbacks of this kind of installation is the limited interaction space for people "using" a public projection: messages are mostly unidirectional and there is a little that a person can actually do to be engaged in a richer interaction than consuming a simple information broadcast.

This work reports on the research aiming at using public art installations to address multiple (previously unconnected) people at the same time, increasing the level of social connectedness among them, and finally evaluating the attractiveness of the installation. The main challenge is to establish the public installation as a social actor - a socially acceptable participant in a social multi-user setting, in which the culture 
would matter [2, 3]. Part of this research is also to investigate whether computers (controlling the public installation) can indeed act as social actors and improve social connectedness. "Social actor", in general ICT uses, was developed into a conceptualization model through a series of empirical studies. There are four dimensions in the conceptualization of a social actor: affiliations, environments, interactions, identities and temporalities [4].

In the field of HCI, computers are considered to be able to handle social tasks and tend to be treated like humans [5]. There is a growing community around public projection and large-scale installations, and social interaction of their users, which is picked up by user-dedicated devices such as RFID tags and mobile phones [6-8]. In the case of Blobulous, an interactive installation to be introduced in the next section, the large (possibly public) projection of abstract avatars is combined with bio signals, i.e., the heart rate, which other research also consider as a reliable and effective means of communication between people $[9,10]$. With the system we explore the possibilities in utilizing related technologies to collect information from wearable objects for social interaction in public spaces [11].

\section{Blobulous System}

Blobulous is a novel interactive installation (see Fig. 1 for example settings and Fig. 2 for system overview) that interacts with participants through projected avatars in public spaces, which react to the participants' movement and body signals. Blobulous uses a large (possible public) projection to show abstract avatars, blobs of dots therefore the name "Blobulous" - one for each participant and moving around slowly. The movement of the avatars is connected to the participant's movement in the space in front of the projection. The second mapping involved in the installation is from a participant's heart rate to the color of his or her avatar. The mapped colors range from blue (cold, low engagement) to red (warm, high engagement).

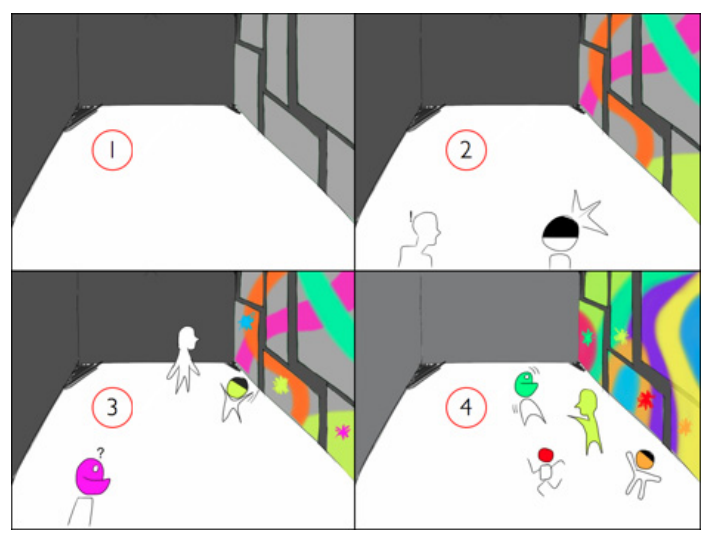

Fig. 1. Example space for using Blobulous 


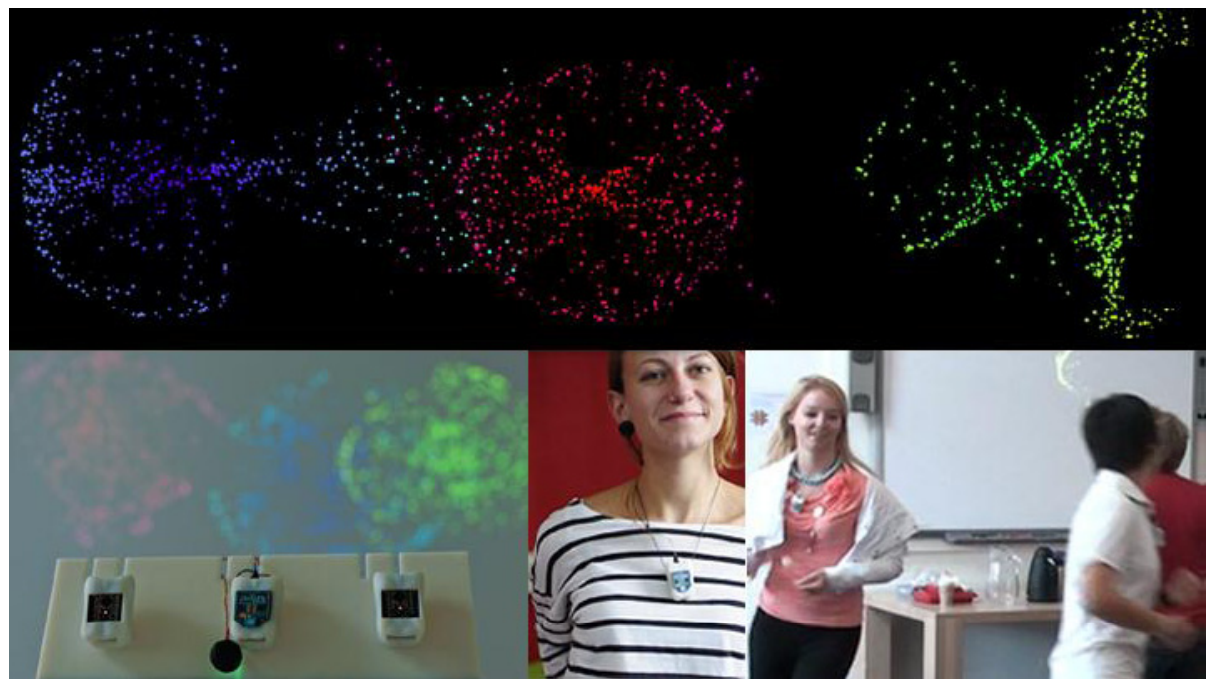

Fig. 2. Overview of system components

The Blobulous system consists of four parts:

1. Wireless heart rate sensors capture and send heart rate data from users to a central instance,

2. a central instance, including a receiver and a visual program, receives data from users and derives avatar behaviors represented as visuals on the projected screen,

3. a projector connected to the central instance, and

4. a Zigbee ${ }^{1}$ network, which handles communication between sensors and the central instance.

\section{Evaluation}

The objective of evaluating the Blobulous system is to show an improvement of social connectedness among participants and the attractiveness of the installation. Social connectedness is measure by means of a questionnaire that has been derived from Social Connectedness Scale Revised (SCS_R) questionnaire [12]. The results about the social connectedness is reported in [13]. The experiment results showed a significant difference in the level of social connectedness between the two testing conditions (random avatars and interactive, mapped avatars).

In this paper we focus on reporting the evaluation of the attractiveness of the installation.

${ }^{1}$ http: / / www . zigbee.org/ 


\subsection{Experiment Setup}

In order to evaluate the attractiveness of the installation, it is better to include a group dynamics factor in the evaluation. 21 (14 male, 7 female) participants were recruited online and randomly divided into 7 groups according to their time preference. So, in most of the groups, participants did not know each other before the experiment. Users' backgrounds were distributed to Industrial Design (7), Electrical Engineering (4), Computer Science (3), Automotive/Logistics (3), Biomedical (2), Architecture (1), and Business (1).

Participants were asked to watch and explore the visuals projected on the wall (Fig. 3a) while wearing the sensor (Fig. 3b) and then have a short discussion about what they perceive from the visuals. Heart rate data was streaming automatically by the prototype while movement data was manually controlled via an Apple iPad using touchOSC [14] (Wizard of Oz) (Fig. 3c).

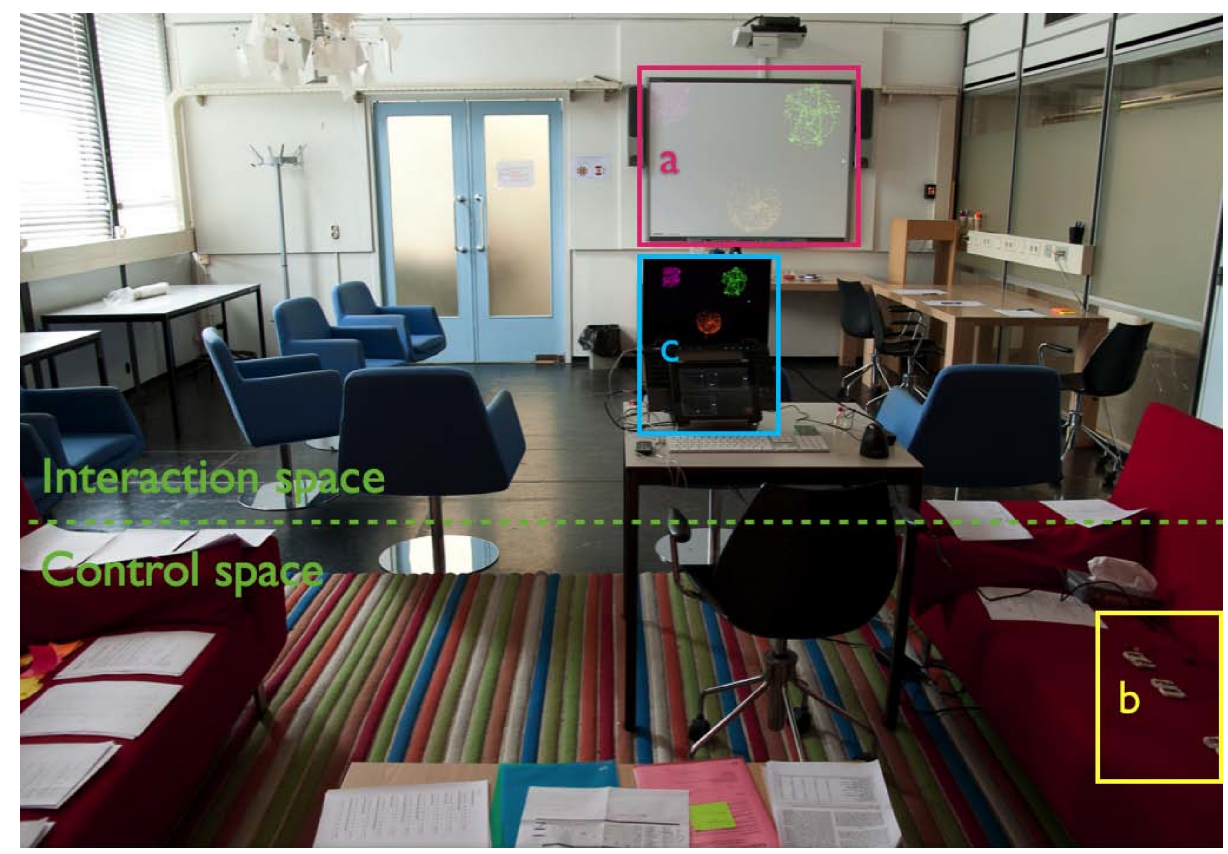

Fig. 3. Experiment room with a) projection screen, b) heart rate sensors, and c) central control

In the demo session, participants were explained details about the functionality of Blobulous and asked to come up with some ideas and try to demonstrate the ideas together with Blobulous. All sessions were recorded for later video analysis. The experiment room was prepared with a large projection onto the wall, an interaction space in front of the projection, and an experiment control area (depicted at the bottom of Fig. 3). 


\subsection{Instrument}

AttrakDiff [15] is an instrument for measuring the attractiveness of interactive products. With the help of pairs of opposite adjectives, users (or potential users) can indicate their perception of the product. These adjective-pairs make a collation of the evaluation dimensions possible.

The following product dimensions are evaluated:

- Pragmatic Quality (PQ): Describe the usability of a product and indicates how successfully users are in achieving their goals using the product.

- Hedonic Quality - Stimulation (HQ-S): Mankind has an inherent need to develop and move forward. This dimension indicates to what extent the product can support those needs in terms of novel, interesting, and stimulation functions, contents, and interaction- and presentation styles.

- Hedonic Quality - Identity (HQ-I): Indicates to what extent the product allows the users to identify with it.

- Attractiveness (ATT): Describes a global value of the product based on the quality perception.

Hedonic and pragmatic qualities are independent of one another, and contribute equally to the rating of attractiveness.

\subsection{Results}

The map in Fig. 4 was generated from the attrakdiff.de web service [15]. In this map, the values of hedonic quality are represented on the vertical axis (bottom $=$ low value).
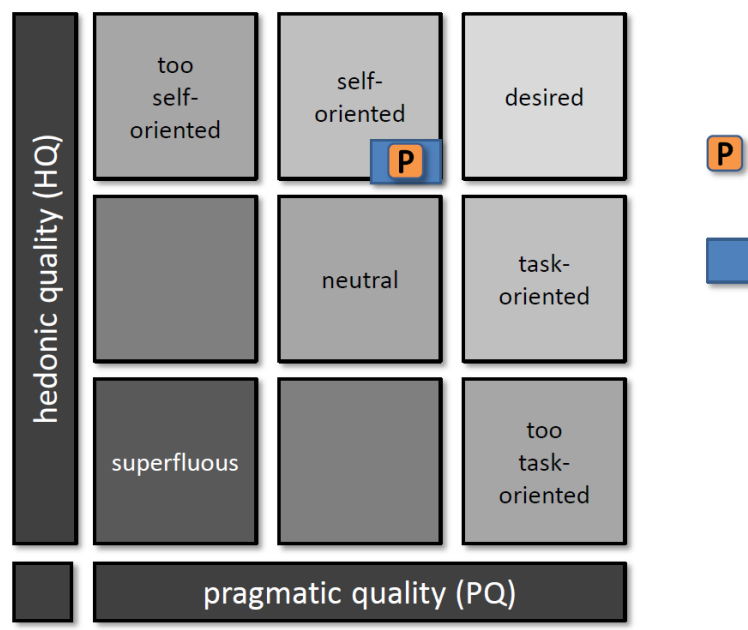

Medium value of the dimensions

with the product Blobulous

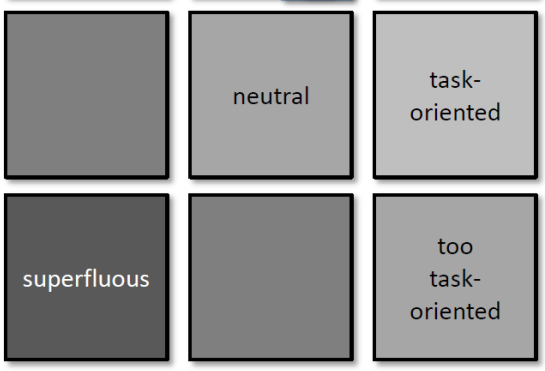

pragmatic quality (PQ)

Fig. 4. Map with average values of the dimensions $P Q$ and HQ and the confidence rectangle 
The horizontal axis represents the value of the pragmatic quality (left $=$ low value). Depending on the dimensions values the installation (called a product by AttrakDiff) will lie in one or more "character-regions". The installation was rated as fairly "selforiented". However the value of pragmatic quality just reaches above the average values. Consequently, there is room for improvement in terms of usability. In terms of hedonic quality, the classification applies positively. The user is stimulated by the prototype; however the hedonic value is again just above average. So there is room for improvement in hedonic quality as well. The confidence rectangle is relatively small which shows the users are aware that they are evaluating a prototype, instead of a real product.

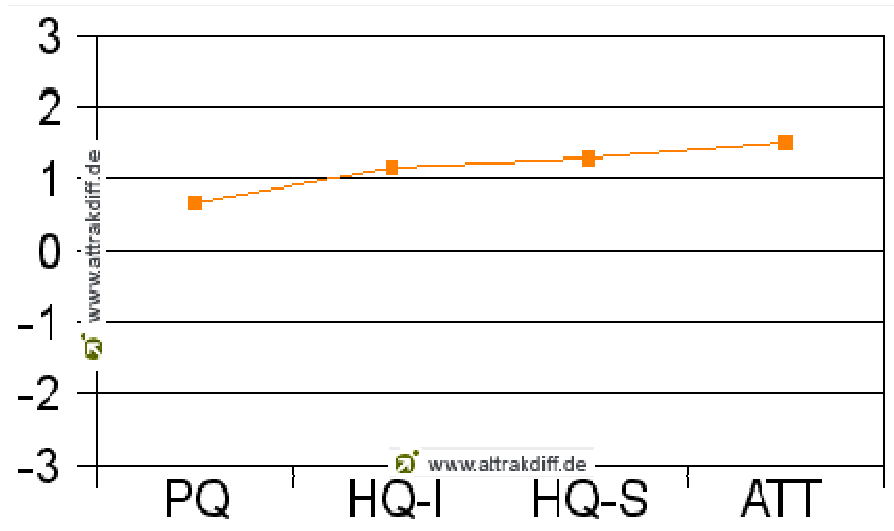

Fig. 5. Mean values of the four AttrakDiff dimensions for the prototype

Fig. 5 features a diagram of average values of the four dimensions PQ, HQ-I, HQ$\mathrm{S}$ and ATT. With regard to HQ-I, the prototype is located in the above-average region. It provides the user with identification and thus meets ordinary standards. In order to bind the users more strongly to the concept, we must aim at improvement in the design. About HQ-S, the prototype is located in the above-average region and meets ordinary standards. In order to motivate and stimulate users even more intensely, we must aim at further improvement. The attractiveness value is located in the above-average region, which suggests the overall impression of the prototype is very attractive to the participants.

Fig. 6 presents the mean values of the word-pairs using in AttrakDiff questionnaire. Of particular interest are the extreme values. These show which characteristics are particularly well-received by the participants. For the Blobulous prototype it is clear that it is perceived as practical, manageable, integrating, bringing me closer, presentable, inventive, creative, innovative, captivating, challenging, novel, pleasant, attractive, likeable, inviting, appealing and motivating, while most of the word-pairs shows a tendency towards a positive experience. 


\section{Description of word-pairs}

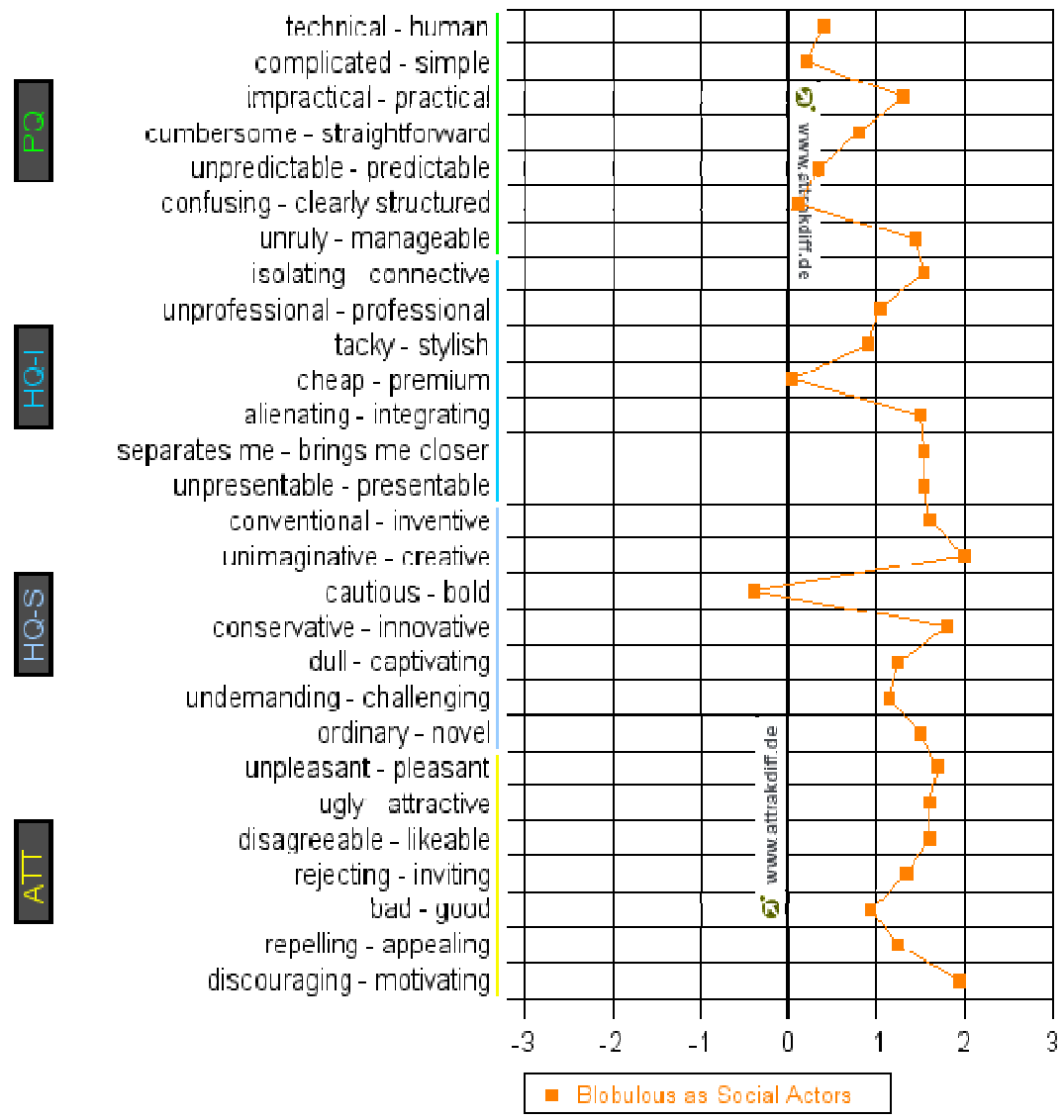

Fig. 6. Mean values of the AttrakDiff word pairs for the prototype

\section{Conclusions and Future Work}

The Blobulous installation was designed to act as a social actor, specifically to improve social connectedness between visitors. Blobulous draws great attention from users due to its colorful appearances and lively movements. It also raises social awareness between people while they are (acting) together. With those effects, Blobulous makes people talk about it, about each other and sometimes they try to understand Blobulous. By connecting people and computers physiologically, Blobulous has clear impact on social interaction and it also shows the attractiveness to people from aspects such as creativity, novelty, inviting and motivating. 
In this work we also found that the AttrakDiff instrument to be useful and convenient in evaluating the attractiveness of public art installations.

The system needs to be further developed with the ability to act independently but not only mimicking to do so, which was a pragmatic design choice in this study. We are currently planning to bring the experience we had with Blobulous in a bigger public art installation project that will be carried out in Taicang, China. We are going to use multiple large LED displays and projections as part of a permanent public art installation, in combination with other art forms such as reliefs, lighting and metal work (see Fig. 7). The content of the displays and projects will be created by general public using social tools over social media ${ }^{2}$.

It is clear that the current development in digital public arts involves a significant amount of new carriers in not only material, but also in technology, resulting new dynamic and interactive forms that require the artists to construct their work from a system view and with a good understanding of human-system interaction and related interface technologies.

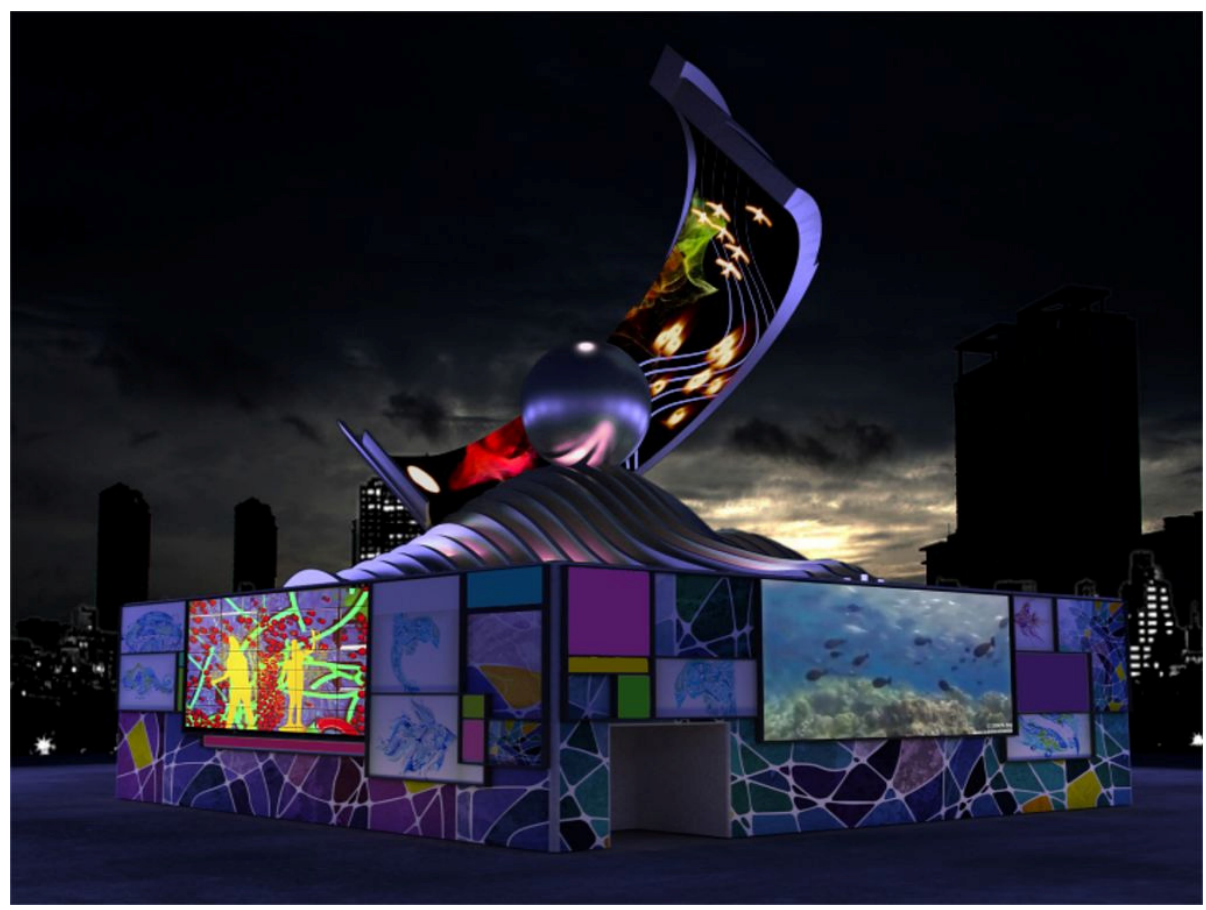

Fig. 7. Interactive public art installation concept for Taicang, China

2 http://youtu.be/m_BjzlekIdI 


\section{References}

1. Wang, F., Hu, J., Rauterberg, M.: New Carriers, Media and Forms of Public Digital Arts. In: Culture and Computing, pp. 83-93. Springer, Heidelberg (2012)

2. Hu, J., Bartneck, C.: Culture Matters - a Study on Presence in an Interactive Movie. CyberPsychology and Behavior 11(5), 529-535 (2008)

3. Hu, J., et al.: ALICE's Adventures in Cultural Computing. International Journal of Arts and Technology 1(1), 102-118 (2008)

4. Lamb, R.: Alternative paths toward a social actor concept. In: Proceedings of the Twelfth Americas Conference on Information Systems. Citeseer (2006)

5. Reeves, B., Nass, C.I.: The media equation: How people treat computers, television, and new media like real people and places. Center for the Study of Language and Information, Cambridge University Press, Chicago, New York (1996)

6. Rogers, Y., Brignull, H.: Subtle ice-breaking: encouraging socializing and interaction around a large public display. In: Workshop on Public, Community. and Situated Displays (2002)

7. Rukzio, E., Wetzstein, S., Schmidt, A.: A Framework for Mobile Interactions with the Physical World. In: Proceedings of Wireless Personal Multimedia Communication, WPMC 2005 (2005)

8. Villar, N., et al.: Interacting with proactive public displays. Computers \& Graphics 27(6), 849-857 (2003)

9. Cwir, D., et al.: Your heart makes my heart move: Cues of social connectedness cause shared emotions and physiological states among strangers. Journal of Experimental Social Psychology 47(3), 661-664 (2011)

10. Slovák, P., Janssen, J., Fitzpatrick, G.: Understanding heart rate sharing: towards unpacking physiosocial space. In: Proceedings of the 2012 ACM Annual Conference on Human Factors in Computing Systems. ACM (2012)

11. van der Vlist, B., et al.: Semantic Connections: Exploring and Manipulating Connections in Smart Spaces. In: 2010 IEEE Symposium on Computers and Communications (ISCC), pp. 1-4. IEEE, Riccione (2010)

12. Lee, R.M., Draper, M., Lee, S.: Social connectedness, dysfunctional interpersonal behaviors, and psychological distress: Testing a mediator model. Journal of Counseling Psychology 48(3), 310-318 (2001)

13. Le, D., Funk, M., Hu, J.: Blobulous: Computers As Social Actors. In: CHI 2013 workshop on Experiencing Interactivity in Public Spaces, EIPS, Paris (2013)

14. Helxer.net. TouchOSC (2012), http: / / hexler. net/software/touchosc

15. User Interface Design $\mathrm{GmbH}$, AttrakDiff Tool to measure the perceived attractiveness of interactive products based on hedonic and pragmatic quality (2012), http: / / www . attrakdiff.de/en/Home/ 